\title{
UV radiative feedback on high-redshift proto-galaxies
}

\author{
Andrei Mesinger, Greg L. Haiman, and Zoltán Haiman \\ Department of Astronomy, Columbia University, \\ 550 West 120th Street, New York, NY 10027, USA
}

\begin{abstract}
We discuss the UV radiative feedback on high-redshift proto-galaxies.
Keywords. cosmology: theory, early Universe, galaxies: high-redshift, evolution
\end{abstract}

We use three-dimensional hydrodynamic simulations to investigate the effects of a transient photo-ionizing ultraviolet (UV) flux on the collapse and cooling of pregalactic clouds. These clouds have masses in the range $10^{5-7} \mathrm{M}_{\odot}$, form at high redshifts $(z \gtrsim 18)$, and are assumed to lie within the short-lived cosmological H II regions around the first generation of stars. In addition, we study the combined effects of this transient UV flux and a persistent Lyman-Werner (LW) background (at photon energies below $13.6 \mathrm{eV}$ ) from distant sources. In the absence of a LW background, we find that a critical specific intensity of $J_{\mathrm{UV}} \simeq 0.1 \times 10^{-21} \mathrm{erg} \mathrm{s}^{-1} \mathrm{~cm}^{-2} \mathrm{~Hz}^{-1} \mathrm{sr}^{-1}$ demarcates a transition from net negative to positive feedback for the halo population. A weaker UV flux stimulates subsequent star formation inside the fossil $\mathrm{H}$ II regions, by enhancing the $\mathrm{H}_{2}$ molecule abundance. A stronger UV flux significantly delays star-formation by reducing the gas density, and increasing the cooling time, at the centers of collapsing halos.

At a fixed $J_{U V}$, the sign of the feedback also depends strongly on the density of the gas at the time of UV illumination. Regardless of whether the feedback is positive or negative, we find that once the UV flux is turned off, its impact starts to diminish after $\sim 30 \%$ of the Hubble time. In the more realistic case when a LW background is present, with $J_{\mathrm{LW}} \gtrsim 0.01 \times 10^{-21} \mathrm{erg} \mathrm{s}^{-1} \mathrm{~cm}^{-2} \mathrm{~Hz}^{-1} \mathrm{sr}^{-1}$, strong suppression persists down to the lowest redshift $(z=18)$ in our simulations.

Finally, we find evidence that heating and photoevaporation by the transient UV flux renders the $\sim 10^{6} \mathrm{M}_{\odot}$ halos inside fossil $\mathrm{H}$ II regions more vulnerable to subsequent $\mathrm{H}_{2}$ photo-dissociation by a LW background.

See Mesinger et al. (2006) for details of our work.

\section{Reference}

Mesinger, A., Bryan, G. L., \& Haiman, Z. 2006, ApJ, 648, 835 